\title{
Search for Chemically Defined Culture Media to Assist Initial Regeneration of Diseased Renal Parenchyma after Stem/Progenitor Cell Implantation
}

\author{
Will W. Minuth ${ }^{1 *}$, Lucia Denk ${ }^{1}$ and Michael Gruber
}

${ }^{1}$ Department of Molecular and Cellular Anatomy, University of Regensburg, Regensburg, Germany

${ }^{2}$ Department of Anesthesiology, University Hospital, Regensburg, Germany

\begin{abstract}
Before an intended implantation, stem/progenitor cells are usually kept in the beneficial atmosphere of a selected culture medium. However, after implantation, the situation is drastically changing for them. Yet stem/progenitor cells must stand the harmful fluid environment within a diseased organ. In this coherence it is unknown, to which degree alterations in molecular composition of interstitial fluid can influence the initial regeneration of parenchyma.

To obtain first insights in the sensitivity against changes in fluid composition, renal stem/progenitor cells were mounted within a polyester interstitium for perfusion culture. To model interstitial fluids different chemically defined culture media all including aldosterone were administered continuously for 13 days. Then morphological quality of generated tubules was registered by light and transmission electron microscopy.

Culture of stem/progenitor cells in earlier approved Iscove's Modified Dulbecco's Medium served as internal standard. These experiments revealed generation of numerous tubules. In comparison, application of Williams' E Medium, Basal Medium Eagle, McCoy's 5A Modified Medium and Medium 199 produced only a lean quality of generated tubules, since contained cells exhibited numerous vacuoles. In contrast, administration of Leibovitz's L-15 Medium and $\mathrm{CO}_{2}$ Independent Medium showed unexpected promoting effects on development of tubules. In this series, numerous and intact tubules without formation of an excess of vacuoles were detected.
\end{abstract}

In consequence, Leibovitz's L-15 Medium and $\mathrm{CO}_{2}$ Independent Medium appear as challenging candidates to be tested in future for implantation in combination with stem/progenitor cells.

Keywords: Kidney; Stem/progenitor cells; Artificial interstitium; Perfusion culture; Chemically defined culture medium

\section{Introduction}

Numerous papers published in the course of the last years demonstrate that a stem/progenitor cell-based therapy appears as an attractive option to cure acute and chronic renal failure in future $[1,2]$. However, critical reading of literature also elucidates that this innovative therapeutic approach is still in an early phase of research and clinical trials [3].

In consequence, until a reliable therapeutic application is available, a series of biomedical problems such as an effective implantation of stem/progenitor cells, compensation of harmful influences derived from interstitial fluid of diseased parenchyma and controlled repair of nephron-specific structures has to be elaborated [4].

Beside the infusion via the blood vessel system [5,6] (Figure 1a) or punctual implantation into the diseased parenchyma [7] (Figure $1 \mathrm{~b})$, an alternative project is to implant stem/progenitor cells between the organ capsule and the outer parenchyma at the earlier site of nephron formation [8] (Figure 1c). However, independent from the kind of surgical application, a crucial problem is that up to harvest stem/progenitor cells are contained in the beneficial atmosphere of a culture medium, while after implantation exposure to the harmful environment of degenerating nephrons, altered extracellular matrix, unbalanced growth factors, interleukins and hormones takes place [9$11]$.

Moreover, the interstitial fluid within diseased parenchyma shows lack of oxygen due to damage of peritubular capillaries and contains a series of harmful metabolites causing inflammation and accelerating further the process of degeneration [12-15]. In such a harmful atmosphere, stem/progenitor cells have to stand and must turn the situation into an environment supporting repair of parenchyma. In consequence, the strategy for implantation is to absorb harmful fluctuations of the interstitial fluid by co-implantation of a suitable buffering fluid $[16,17]$.

A technical solution might be to mount in a first step stem/ progenitor cells together with a potent buffering culture medium within an artificial polyester interstitium [18]. In a second step, the construct can be implanted under the kidney capsule. In this scenario, a mechanical protection of stem/progenitor cells will be maintained by the fleece fibers, while the space between the fibers acts as an extended reservoir of fluid so that at least for the initial period of repair contained stem/progenitor cells are protected from harmful interstitial fluid of diseased parenchyma.

When implantation by the help of an artificial interstitium is considered, a pivotal role in the process of regeneration plays the coimplanted culture medium. It is contained in the space between the fleece fibers, compensates environment and provides stem/progenitor cells with nutrition and respiratory gas [19]. In this special case, the

*Corresponding author: Will W. Minuth, Molecular and Cellular Anatomy, University of Regensburg, Universitystreet 31, D-93053 Regensburg, Germany, Tel: +49 941 443 2876; Fax: +49 941943 2868; E-mail: will.minuth@vkl.uni-regensburg.de

Received January 07, 2013; Accepted February 12, 2013; Published February 15, 2013

Citation: Minuth WW, Denk L, Gruber M (2013) Search for Chemically Defined Culture Media to Assist Initial Regeneration of Diseased Renal Parenchyma after Stem/Progenitor Cell Implantation. J Tissue Sci Eng 4: 123. doi:10.4172/21577552.1000123

Copyright: (C) 2013 Minuth WW, et al. This is an open-access article distributed under the terms of the Creative Commons Attribution License, which permits unrestricted use, distribution, and reproduction in any medium, provided the original author and source are credited. 
Citation: Minuth WW, Denk L, Gruber M (2013) Search for Chemically Defined Culture Media to Assist Initial Regeneration of Diseased Renal Parenchyma after Stem/Progenitor Cell Implantation. J Tissue Sci Eng 4: 123. doi:10.4172/2157-7552.1000123

Page 2 of 7
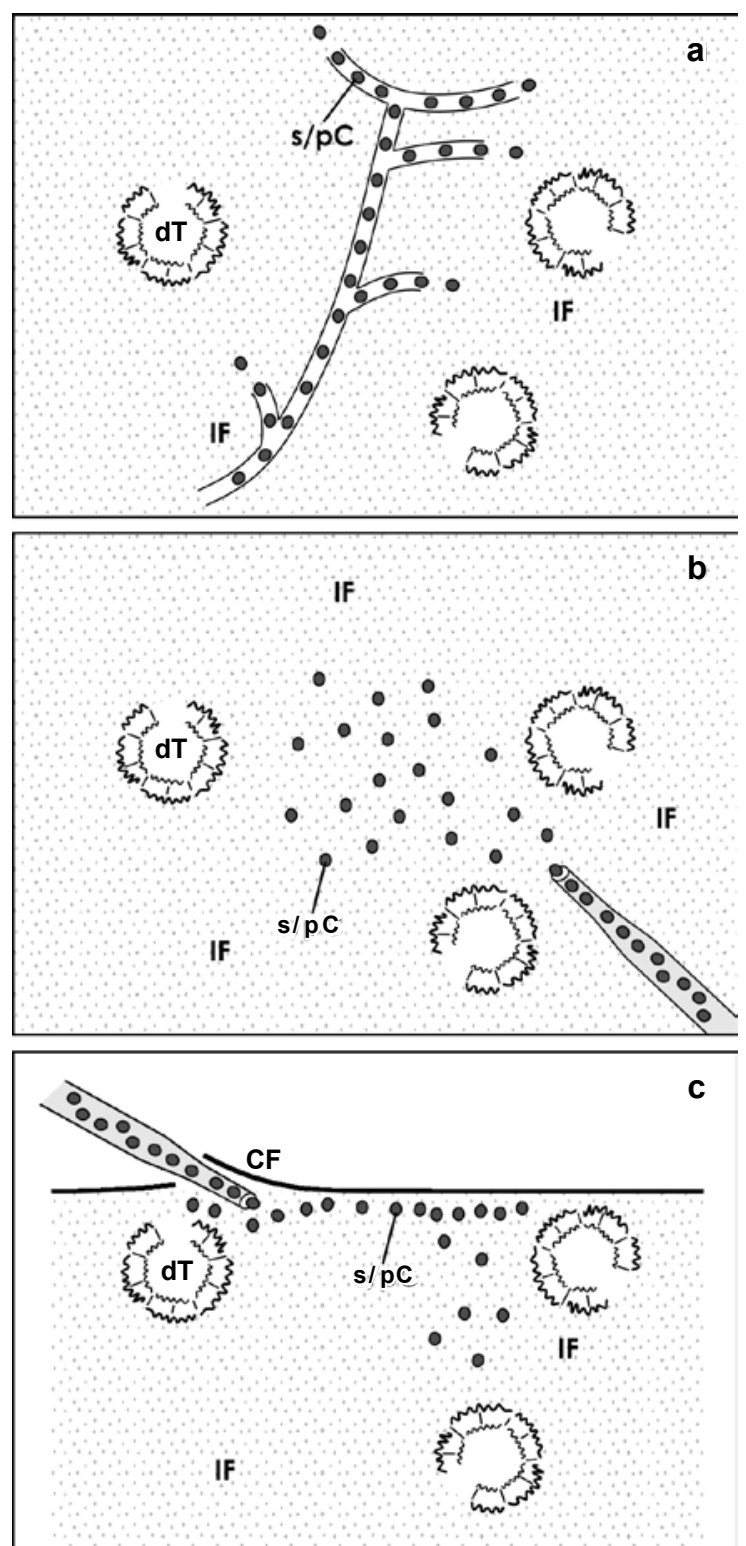

Figure 1: Schematic illustration presents techniques for implantation of stem/ progenitor cells (s/pC, grey dot) into a diseased kidney. (a) As well after infusion via the blood vessel system as after; (b) punctual implantation into diseased parenchyma and (c) injection between the capsula fibrosa (CF) and the outer parenchyma stem/progenitor cells are exposed to the harmfu interstitial fluid (IF) and damaged tubules (dT).

volume of contained fluid must be big enough to support survival, multiplication, potency and development of stem/progenitor cells. Moreover, the available culture medium has to equilibrate the instability of $\mathrm{pH}$ and the influences of unbalanced metabolites of surrounding interstitial fluid in diseased parenchyma.

It is well known that commercially available culture media exhibit a wide range of electrolyte compositions and buffer systems. However, regarding implantation of stem/progenitor cells in combination with initial repair of parenchyma, it is not known which special sort of medium appears as the most suitable one [20-23]. Thus, for gathering more experiences regarding renal tubule regeneration and for finding appropriate candidates the present experiments were performed.

\section{Materials and Methods}

\section{Isolation of renal stem/progenitor cells}

From one-day old anesthesized and sacrificed New Zealand rabbits (Seidl, Oberndorf, Germany), both kidneys were removed under sterile conditions and cut into a ventral and dorsal half as earlier described $[18,19]$. By stripping off the capsula fibrosa with fine forceps, a thin layer of stem/progenitor cell niches is adherent to the explant. When this simple isolation method is performed, an embryonic tissue layer of up to $1 \mathrm{~cm}^{2}$ in square can be harvested.

Perfusion culture: Renal embryonic tissue containing numerous renal stem/progenitor niches was mounted within a polyester interstitium and cultured for 13 days in chemically defined culture media to evaluate their influence on tubule development. Briefly, the isolated tissue layer was placed between two punched out pieces of polyester fleece resulting in a sandwich set-up (I7, Walraf, Grevenbroich, Germany) [18]. For mounting a polyester fleece measuring $13 \mathrm{~mm}$ in diameter was placed inside a Minusheet tissue carrier (Minucells and Minutissue, Bad Abbach, Germany). Then the sandwich set-up containing renal stem/progenitor cells was inserted. Finally, another fleece was placed on top. Then the tissue carrier was transferred to a perfusion culture container with horizontal flow characteristics. For a period of 13 days, fresh medium was continuously transported at a rate of $1.25 \mathrm{ml} / \mathrm{h}$ with an IPC N8 peristaltic pump (Ismatec, Wertheim, Germany).

Applied culture media: To generate tubules chemically defined Williams' E Medium (Nr. 32551-020), Basal Medium Eagle (BME, Nr. 41010-026), McCoy's 5A Modified Medium (Nr. 22330-021), Iscove's Modified Dulbecco's Medium (IMDM, Nr. 21980-032), Medium 199 (Nr. 31153-026), Leibovitz's L-15 Medium (Nr. 31415-029) and $\mathrm{CO}_{2}$ Independent Medium (Nr. 18045-054) all including phenol red were applied. All media were obtained from GIBCO/Invitrogen, Karlsruhe, Germany. Infections were prevented by adding an antibiotic-antimycotic cocktail (1\%, GIBCO/Invitrogen). Tubulogenic development was induced by application of aldosterone $\left(1 \times 10^{-7} \mathrm{M}\right.$, Fluka, Taufkirchen, Germany) as earlier described $[18,19]$. To reach a constant $\mathrm{pH}$ of 7.4 under atmospheric air 4-(2-hydroxyethyl) piperazine-1-ethanesulfonic acid (HEPES; GIBCO/Invitrogen) was added during titration in necessary amounts.

Determination of electrolyte and glucose content was performed using a plasma calibrated Bloodgas Analyser ABL800 FLEX Series (Radiometer $\mathrm{GmbH}$, Willich, Germany) according manufacturer's instructions.

Histochemistry on cultures: After run of perfusion culture experiments the sandwich set-ups containing renal parenchyma within layers of polyester fleece ( $5 \mathrm{~mm}$ diameter) were embedded in $1 \%$ agarose (Serva, Heidelberg, Germany), then surrounded by TissueTek (O.C.T. ${ }^{\mathrm{TM}}$ COMPOUND, Sakura Finetek, Zoeterwoude, Netherlands) and frozen at $-80^{\circ} \mathrm{C}$. To analyze cell biological features $20 \mu \mathrm{m}$ thick cryosections were made and stained for control with Toluidine blue or labeled with fluorescent Soybean Agglutinin (SBA, Vector, Burlingame, USA) as earlier described [19].

Transmission electron microscopy: To investigate semithin and ultrathin sections of generated tubules, specimens were transferred to immersion fixation in 5\% glutaraldehyde (Serva) buffered with $0.15 \mathrm{M}$ sodium cacodylate, $\mathrm{pH}$ 7.4. Fixation was performed for 1 day at room temperature. After several washes with $0.15 \mathrm{M}$ sodium cacodylate, the specimens were postfixed in the same buffer but containing additionally 
$1 \%$ osmium tetroxide (Science Services, München, Germany). Then the tissue was rinsed with sodium cacodylate buffer and dehydrated in graded series of ethanols. Finally, specimens were embedded in Epon (Fluka) polymerizing at $60^{\circ} \mathrm{C}$ for $48 \mathrm{~h}$.

Semithin and ultrathin sections were made with a diamond knife on an ultramicrotome Ultracut E (Reichert-Jung, Nußloch, Germany). Semithin sections were labeled with Richardson solution and then analyzed using an Axioskop 2 plus (Zeiss, Oberkochen, Germany). Images were taken with a digital camera at a $250 \mathrm{~ms}$ standard exposure time and thereafter processed with Corel DRAW Graphic Suite X5 (Corel Corporation, Otawa, Canada).

Ultrathin sections were collected onto slot grids (Plano, Wetzlar, Germany) coated with $1.5 \%$ pioloform (Plano) and contrasted using $2 \%$ uranyl acetate and lead citrate (Fluka) as earlier described [24].

\section{Definition of cells within the renal stem/progenitor cell niche}

For the presented experiments, embryonic tissue derived from the outer cortex of neonatal rabbit kidney was analyzed containing renal stem/progenitor cell niches. To avoid confusion, the nomenclature of previously published papers was used $[24,25]$.

\section{Related ethical rules/amount of analyzed specimens}

Performed experiments are in accordance with the Animal Ethics Committee, University of Regensburg, Regensburg, Germany. For the present investigation, a total of 50 generated specimens and in each series, at least 3 individual samples were morphologically analyzed. In all micrographs, the typical biological mean is represented.

\section{Results}

\section{Basic composition of applied culture media}

To simulate regeneration under advanced culture conditions, renal stem/progenitor cells were mounted for present experiments in an artificial polyester interstitium. For obtaining information about their sensitivity towards alterations in fluid composition, a selection of media was offered during run of perfusion culture experiments. For example, as shown in table 1 , analysis of electrolytes demonstrates that the basic composition of applied culture media is very heterogenous.

According to performed analysis, $\mathrm{CO}_{2}$ Independent Medium illustrates the highest $\mathrm{Na}^{+}$concentration $(159.3 \mathrm{mmol} / \mathrm{L})$, while Medium 199 exhibits with $145.0 \mathrm{mmol} / \mathrm{L}$ the lowest $\mathrm{Na}^{+}$content. Leibovitz's L-15 Medium presents with $148.4 \mathrm{mmol} / \mathrm{L}$ the highest $\mathrm{Cl}$ content and Iscove's Modified Dulbecco's Medium (IMDM) shows with $65.6 \mathrm{mmol} / \mathrm{L}$ the lowest $\mathrm{Cl}^{-}$concentration.

The highest $\mathrm{K}^{+}$concentration with $5.9 \mathrm{mmol} / \mathrm{L}$ has Leibovitz's L-15 Medium, while Iscove's Modified Dulbecco's Medium demonstrates with $3.4 \mathrm{mmol} / \mathrm{L}$ the lowest $\mathrm{K}^{+}$load. The $\mathrm{Ca}^{2+}$ concentration is high in Medium $199(1.7 \mathrm{mmol} / \mathrm{L})$, while rather low with $0.5 \mathrm{mmol} / \mathrm{L}$ in $\mathrm{CO}_{2}$ Independent Medium.
Glucose is present in various concentrations. The highest glucose content with $287.3 \mathrm{mg} / \mathrm{dL}$ was detected in Iscove's Modified Dulbecco's Medium IMDM. In contrast, while the lowest amount of glucose with $5.6 \mathrm{mg} / \mathrm{dL}$ was in Leibovitz's L-15 Medium.

\section{Influence of culture media on development}

After 13 days of perfusion culture, cryosections were made to analyze spatial growth of regenerated tubules. Label with Soybean Agglutinin (SBA) depicts that numerous tubules are developing between the polyester fibers of the artificial interstitium (Figures 2a-g). Culture of specimens as well in Williams' E Medium (Figure 2a) as in Basal Medium Eagle (BME, Figure 2b), McCoy's 5A Modified Medium (Figure 2c), Medium 199 (Figure 2d), Iscove's Modified Dulbecco's Medium (Figure 2e), Leibovitz's L-15 Medium (Figure 2f) or $\mathrm{CO}_{2}$ Independent Medium (Figure 2g) demonstrates that tubules exhibit a lumen and that they are covered by a continuously developed basal lamina. In so far the specimens illustrate that tubules can principally regenerate in the presence of all applied culture media.

\section{Light microscopical analysis}

In the next set of experiments, semithin sections were made to obtain more detailed information about morphological features of generated tubules (Figures 2a-g). Also this series of experiments shows that all of the generated tubules exhibit a basal lamina and a lumen. However, comparison of analyzed tubules demonstrates that differences in morphological quality of tubule cells can be recognized. Application of Williams' E Medium (Figure 2a), Basal Medium Eagle (Figure 2b) and McCoy's 5A Modified Medium (Figure 2c) illuminates that numerous vacuoles are detected in the cytoplasm of tubule cells. This result points out that probably toxic effects or non appropriate compounds within a culture medium interfere the developmental process of tubules during regeneration.

In experiments with Medium 199 (Figure 2d) and earlier approved Iscove's Modified Dulbecco's Medium (Figure 2e) some of the regenerated tubule cells show vacuoles within the cytoplasm, while in the majority of cases, enlarged amount of vacuoles is missing [24] In contrast, the use of Leibovitz's L-15 Medium (Figure 2f) or $\mathrm{CO}_{2}$ Independent Medium (Figure $2 \mathrm{~g}$ ) leads to tubules with fully intact morphology and an inconspicuously looking cytoplasm. Semithin sections of specimens further exhibit that application of all tested media results in tubules with an integrated polarized epithelium. In all of analyzed samples, the luminal side faces a lumen while the basal side rests on a continuously developed basal lamina consisting of a lamina rara, lamina densa and lamina fibroreticularis.

Independently, if tubules exhibit numerous vacuoles (Figures 2a-c) or an inconspicuously looking cytoplasm (Figures 2d-g), an intact tight junctional complex was developed between the luminal and lateral cell sides. Most interestingly, in the case enlarged amount of vacuoles in the cytoplasm is present, the plasma membrane, nucleus and mitochondria appear to be intact. This is a sign that cells are still alive but are obviously kept under suboptimal culture medium conditions.

\begin{tabular}{|c|c|c|c|c|c|c|c|}
\hline Parameters & Williams' E & BME & McCoys 5A & Medium 199 & IMDM & Leibovitz's L-15 & $\mathrm{CO}_{2}$ Independent Medium \\
\hline $\mathrm{Na}^{+}[\mathrm{mmol} / \mathrm{L}][\mathrm{mmol} / \mathrm{L}]$ & 156.4 & 153.7 & 155.9 & 145.0 & 151.5 & 152.7 & 159.3 \\
\hline $\mathrm{Cl}-[\mathrm{mmol} / \mathrm{L}]$ & 121.1 & 120.3 & 109.3 & 126.8 & 65.6 & 148.4 & 115.2 \\
\hline $\mathrm{K}^{+}[\mathrm{mmol} / \mathrm{L}]$ & 4.9 & 4.9 & 5.0 & 5.2 & 3.4 & 5.9 & 4.4 \\
\hline $\mathrm{Ca}^{++}[\mathrm{mmol} / \mathrm{L}]$ & 1.5 & 1.5 & 0.8 & 1.7 & 1.0 & 1.2 & 0.5 \\
\hline Glucose[mg/dL] & 178.3 & 92.9 & 262.9 & 98.9 & 287.3 & 5.6 & 81.6 \\
\hline
\end{tabular}

Table 1: Electrolytes and glucose in applied Williams' E Medium, Basal Medium Eagle (BME), McCoy's 5A Modified Medium, Medium 199, Iscove's Modified Dulbecco's Medium (IMDM), Leibovitz's L-15 Medium and $\mathrm{CO}_{2}$ Independent Medium. Analysis was performed after addition of HEPES, aldosterone and an antibiotic-antimycotic cocktail. In all of the cases, the mean of 5 measures is given. 
Citation: Minuth WW, Denk L, Gruber M (2013) Search for Chemically Defined Culture Media to Assist Initial Regeneration of Diseased Renal Parenchyma after Stem/Progenitor Cell Implantation. J Tissue Sci Eng 4: 123. doi:10.4172/2157-7552.1000123

\section{Electron microscopical view}

Transmission electron microscopy further depicts that promising results were obtained in experiments with Medium 199 (Figure 3a) and Iscove's Modified Dulbecco's Medium (Figure 3b). Although small, medium and big-sized vacuoles are contained in the cytoplasm, the cells are fully polarized and appear healthy. Astonishingly, most impressive results were seen in series with $\mathrm{CO}_{2}$ and $\mathrm{NaH}_{2} \mathrm{CO}_{3}$ independent media. Neither culture with Leibovitz's L-15 Medium (Figure 3c) nor $\mathrm{CO}_{2}$ Independent Medium (Figure 3d) exhibited vacuoles in the cytoplasm of cells so that a perfect appearance of tubules is recognized. In all of the cases the luminal side is in contact with a lumen, while the basal
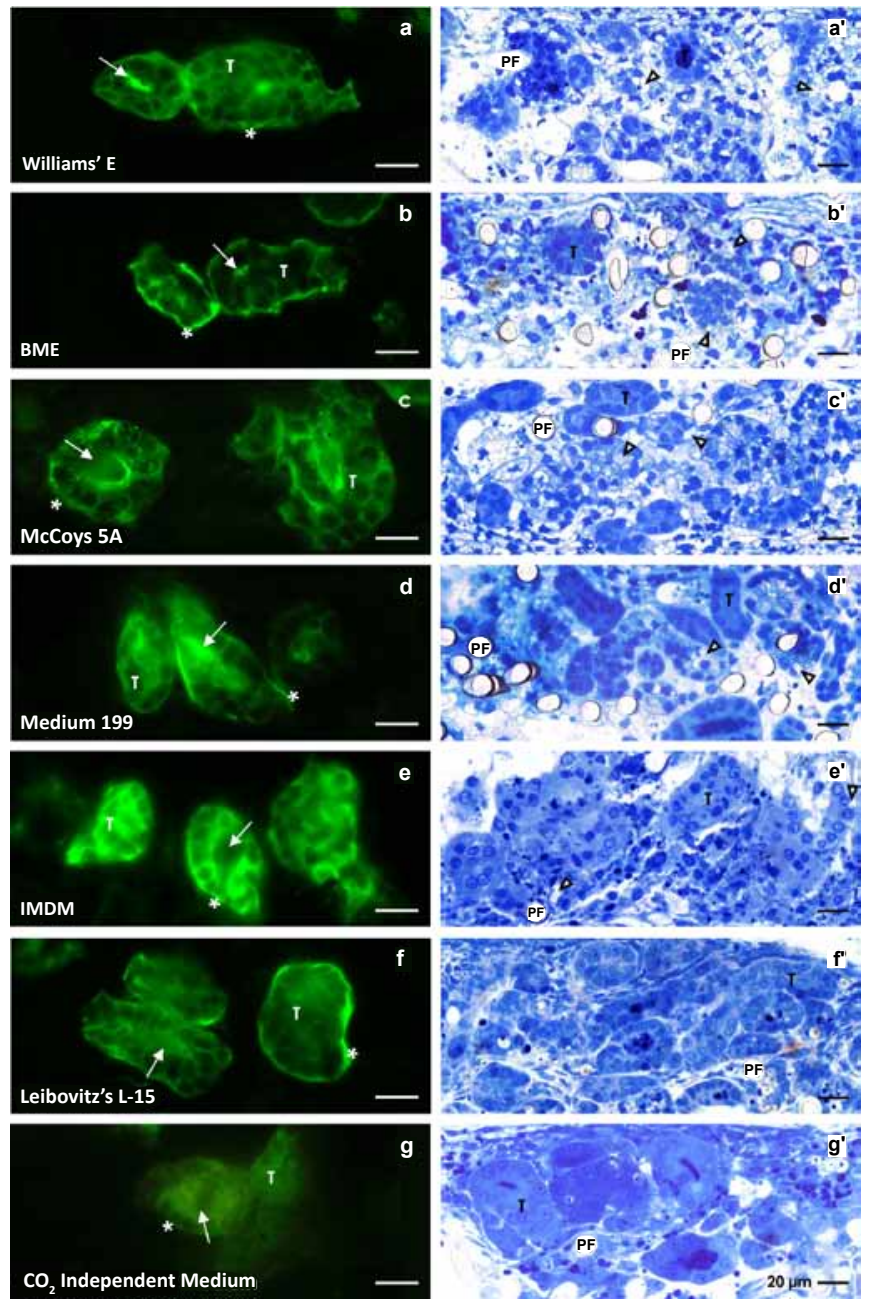

Figure 2: Fluorescent SBA label on cryosections of generated tubules (T) kept in perfusion culture for 13 days. Culture was performed by (a) Williams' E Medium, (b) Basal Medium Eagle (BME), (c) McCoy's 5A Modified Medium, (d) Medium 199, (e) Iscove's Modified Dulbecco's Medium (IMDM), (f) Leibovitz's L-15 Medium and (g) $\mathrm{CO}_{2}$ Independent Medium. In all of the cases, intact tubules are recognized exhibiting a lumen (arrow), an intact epithelium and a covering basal lamina (asterisk). Semithin sections of generated tubules $(T)$ cultured in different chemically defined media for 13 days. Many vacuoles labeled by a lighted arrow head are found in series with (a') Williams' E Medium; (b') Basal Medium Eagle and (c') McCoy's 5A Modified Medium. Few vacuoles can be detected in series with (d') Medium 199 and (e') Iscove's Modified Dulbecco's Medium. In contrast, perfect morphology without vacuoles is obtained after run with (f') Leibovitz's L-15 Medium and (g') $\mathrm{CO}_{2}$ Independent Medium. PF: Polyester fibers.
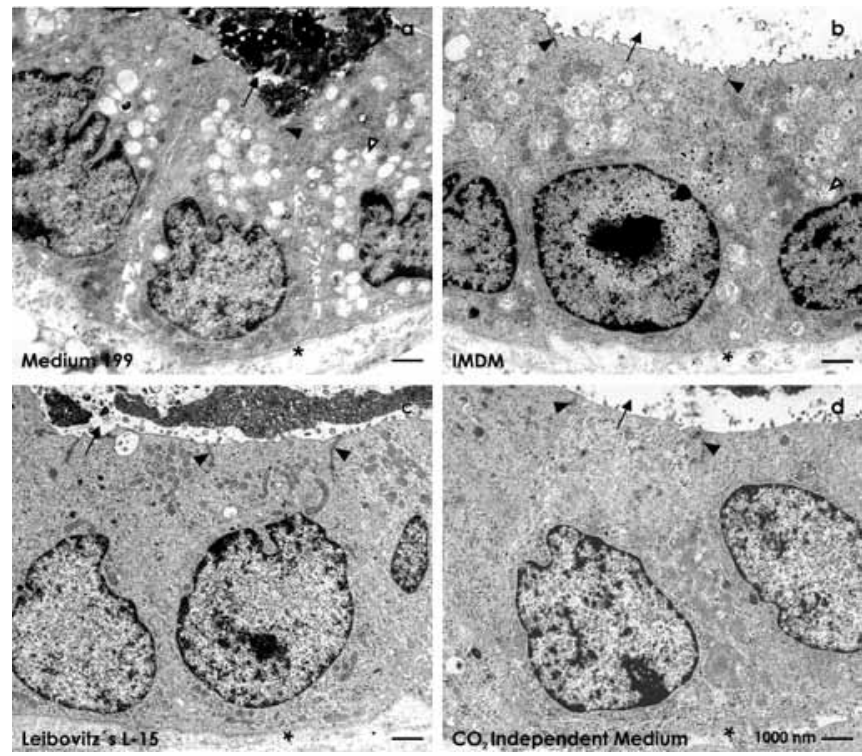

Figure 3: Transmission electron microscopy of epithelial cells within regenerated tubules after 13 days of perfusion culture. In each of the cases, the luminal plasma membranes borders a lumen (arrow), while the lateral membranes are separated by an intact tight junctional complex (black arrow head). The basal membrane is in contact with a basal lamina (asterisk). A low degree of vacuoles can be seen in series with (a) Medium 199 and (b) Iscove's Modified Dulbecco's Medium (IMDM). In contrast, perfect morphology without arise of numerous vacuoles within the cytoplasm is registered after run with (c) Leibovitz's L-15 Medium and (d) $\mathrm{CO}_{2}$ Independent Medium. Vacuoles are labeled by a lighted arrow head.

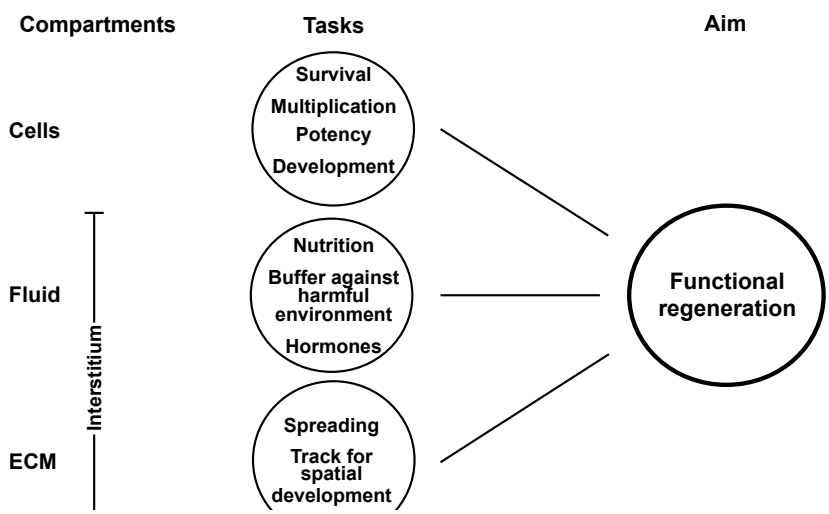

Figure 4: Schematic illustration of requested tasks of stem/progenitor cells during functional regeneration. ECM: Extracellular matrix.

side rests on a continuously developed basal lamina. Finally, between the luminal and lateral plasma membrane an intact tight junctional complex is developed speaking for a perfect physiological sealing between neighboring cells. All of these features speak in favor that tubules can optimally regenerate in Leibovitz's L-15 Medium (Figure $3 \mathrm{c}$ ) and $\mathrm{CO}_{2}$ Independent Medium (Figure 3d).

\section{Discussion}

\section{Protecting implanted cells by a biophysical fence}

The environmental situation alters drastically when stem/progenitor cells are transferred during implantation from the in vitro environment 
Citation: Minuth WW, Denk L, Gruber M (2013) Search for Chemically Defined Culture Media to Assist Initial Regeneration of Diseased Renal Parenchyma after Stem/Progenitor Cell Implantation. J Tissue Sci Eng 4: 123. doi:10.4172/2157-7552.1000123

Page 5 of 7
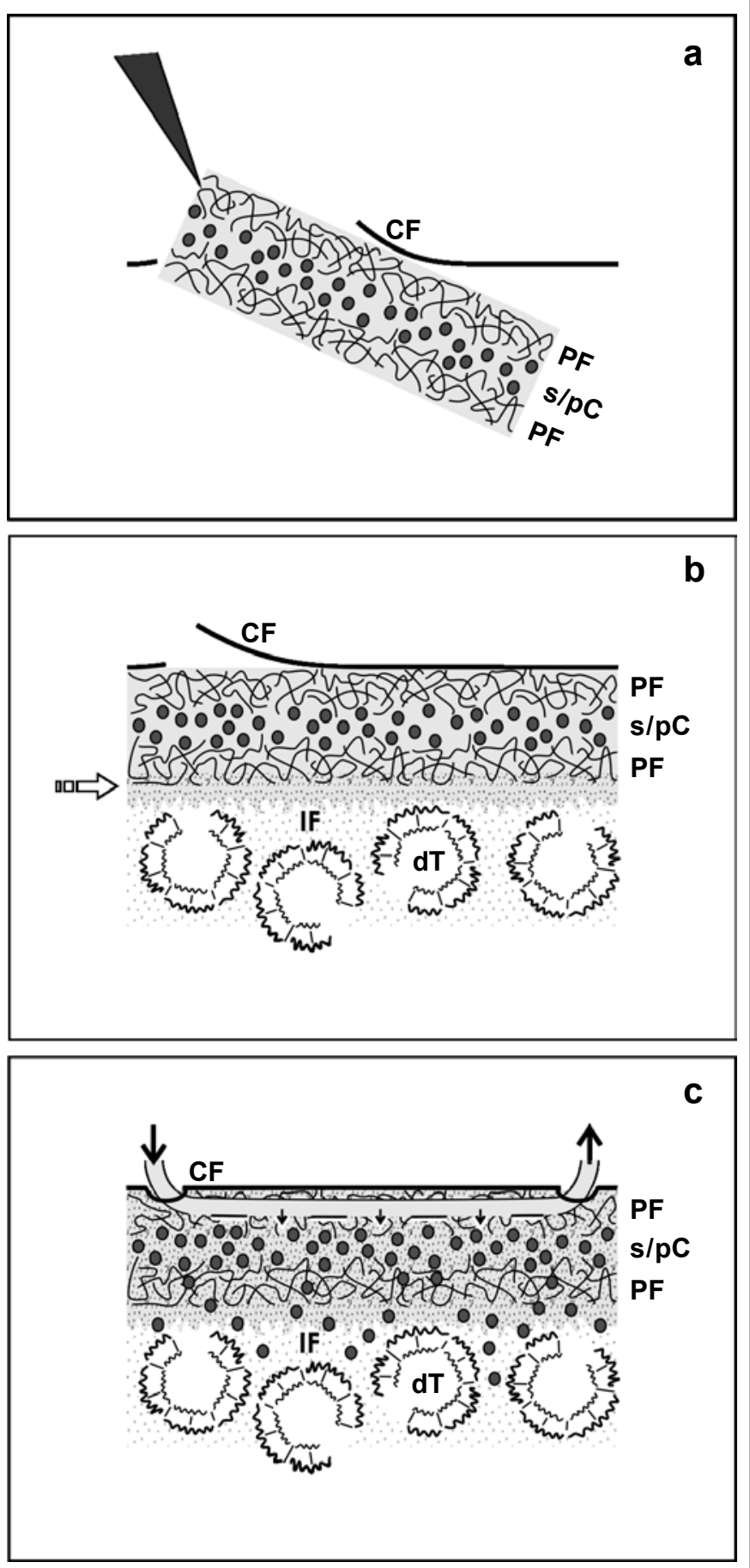

Figure 5: Schematic illustration shows implantation of stem/progenitor cells (s/pC) under the renal capsula fibrosa (CF) after mounting within a polyester fleece (PF). (a) The polyester fibers provide on the one hand mechanical stability during surgical handling. (b) On the other hand, the space between the fleece fibers contains a certain amount of culture medium enabling contained stem/progenitor cells to stand (lighted arrow) influences of harmful interstitial fluid (IF) derived from degenerating tubules (dT). (c) During the initial period of seeding, fresh culture medium can be transported (dark arrow) over a thin tube to the polyester fleece reservoir, while by an efferent tube waste liquid is eliminated. to a diseased kidney $[26,27]$. In a culture dish, stem/progenitor cells are still exposed to a medium stimulating survival, multiplication, potency and development due to contained electrolytes, hormones, buffering substances and nutritional compounds (Figure 4). However, after an implantation, the comfortable environment alters, since they meet harmful interstitial fluid derived from diseased parenchyma. In this critical situation, speading stem/progenitor cells are further confronted with degrading nephron structures, destroyed peritubular capillaries, modified extracellular matrix (ECM) and toxic metabolites [28,29]. Exposed to a more or less chaotic surrounding, stem/progenitor cells must start here to fulfill pleiotropic tasks of development so that they can survive and find the site of primary repair for turning degradation into a process of functional regeneration.

A technical solution might be to seed stem/progenitor cells within a fleece that is implanted under the organ capsule as it was proposed earlier (Figure 5a) $[18,19]$. Such an artificial interstitium consisting of polyester fibers provides on the one hand mechanical stability during surgical handling. Further it exhibits positive interactions with stem/ progenitor cells promoting survival and spatial regeneration of tubules. On the other hand, the space between the fleece fibers contains a certain amount of liquid. In consequence, due to endogenous capillary forces within the fleece, a reservoir for culture medium is present protecting stem/progenitor cells as a biophysical fence against immediate dilution with harmful interstitial fluid.

It is obvious that the contained volume of beneficial culture medium is small so that with ongoing time harmful interstitial fluid will replace it by diffusion (Figure 5b). However, by altering the vastness of the space between the fleece fibers, their diameter or density and the contained volume for culture medium can be varied so that the exchange of contained culture medium with harmful interstitial fluid can be more or less retarded.

Finally, during the initial period of parenchyma repair, fresh culture medium can be transported over a tube to the implanted polyester reservoir, while by an efferent tube waste liquid is eliminated as it is performed in clinic routine by a miniaturized drainage (Figure 5c) [30].

\section{Selecting an optimal fluid environment}

Regarding destroyed structures in diseased renal parenchyma especially the instability of $\mathrm{pH}$ and variations in molecule composition of the interstitial fluid, there is a critical influence on restoration [29]. To obtain first information about the tolerance of stem/progenitor cells against alterations in interstitial fluid, in the present experiments the influence of various culture media such as Williams' E Medium (Figures 2a and 2a'), Basal Medium Eagle (Figures 2b and 2b'), McCoy's 5A Modified Medium (Figures 2c and 2c'), Medium 199 (Figures 2d, $2 \mathrm{~d}^{\prime}$ and 3a), Iscove's Modified Dulbecco's Medium (Figures 2e, 2e' and $3 \mathrm{~b}$ ), Leibovitz's L-15 (Figures $2 \mathrm{f}, 2 \mathrm{f}$ and $3 \mathrm{c}$ ) and $\mathrm{CO}_{2}$ Independent Medium (Figures 2g, 2g' and 3d) on the spatial regeneration of renal tubules within a polyester interstitium was tested.

As illustrated by histochemical label, a first interesting result was that application of all tested culture media is leading to development of tubules. Further fluorescence microscopy demonstrates that in each of the series generated tubules exhibit a basal lamina and a polarized epithelium (Figures $2 \mathrm{a}-\mathrm{g}$ ). Also, analysis of semithin sections demonstrates that it is principally possible to generate tubules by the administration of all tested media (Figures 2a-g). However, these data also illustrate that the quality of generated tubules is different, when mentioned culture media are administered. Although frequently applied in other cell systems, the use of Williams' E Medium (Figure 2a), 
Citation: Minuth WW, Denk L, Gruber M (2013) Search for Chemically Defined Culture Media to Assist Initial Regeneration of Diseased Renal Parenchyma after Stem/Progenitor Cell Implantation. J Tissue Sci Eng 4: 123. doi:10.4172/2157-7552.1000123

Page 6 of 7

Basal Medium Eagle (Figure 2b) and McCoy's 5A Modified Medium (Figure 2c) illustrates that generated tubule cells contain numerous vacuoles within the cytoplasm obviously indicating a toxic influence, suboptimal nutrition or a non-appropriate electrolyte composition. Generation of tubules in Medium 199 (Figures 2d and 3a) and Iscove's Modified Dulbecco's Medium (Figures 2e' and 3b) illustrates that on the one hand tubules exhibit a perfect cytoplasm, while on the other hand some tubules are found containing to a high degree vacuoles within the cytoplasm.

Up-to-date rise of illustrated vacuoles cannot be explained, since the contact to neighbor cells is closed by intact tight junctional complexes. Further, a consistently developed basal lamina is registered. These arguments suggest that it's not a toxic effect but probably nonequilibrated osmolarity or suboptimal nutritional compounds are leading to vacuoles which arise simultaneously.

Best results were obtained by application of Leibovitz's L-15 and $\mathrm{CO}_{2}$ Independent Medium. As seen in semithin sections (Figures $2 \mathrm{f}$ and $2 \mathrm{~g}$ ) and in electron microscopy (Figures $3 \mathrm{c}$ and $3 \mathrm{~d}$ ), all of generated tubules exhibit perfect morphology and an extremely low degree of cytoplasmatic vacuoles. So far the use of Leibovitz's L-15 and $\mathrm{CO}_{2}$ Independent Medium results in an earlier not observed quality of regenerated tubules.

\section{Promoting stem/progenitor cells with $\mathrm{CO}_{2}$ independent media}

Although all chemically defined media allowed the development of renal tubules, differences in quality were observed. The presented experiments illustrate for the first time bad (Figures 2a-2c), mediocre (Figures $2 \mathrm{~d}$ and $2 \mathrm{e}$ ) and optimal (Figures $2 \mathrm{f}$ and $2 \mathrm{~g}$ ) influences of chemically defined culture media on the development of renal stem/ progenitor cells. Surprisingly, the best results were obtained not by earlier used Iscove's Modified Dulbecco's Medium (Figures 2e and 3b) [19] but after application of Leibovitz's L-15 (Figures $2 \mathrm{f}$ and $3 \mathrm{c}$ ) and $\mathrm{CO}_{2}$ Independent Medium (Figures $2 \mathrm{~g}$ and $3 \mathrm{~d}$ ).

According to manufacture's instruction, L-15 Medium is buffered by its complement of salts, free base amino acids and galactose instead of glucose, while the $\mathrm{CO}_{2}$ Independent Medium is buffered by monoand dibasic sodium phosphate and $\beta$-glycerophosphate. Thus, both of these media are not so sensitive in respect to surrounding $\mathrm{NaH}_{2} \mathrm{CO}_{3}$ and $\mathrm{CO}_{2}$ as compared to Williams' E Medium (Figures 2a and 2a'), Basal Medium Eagle (Figures 2b and 2b'), McCoy's 5A Modified Medium (Figures 2c and 2c'), Medium 199 (Figures 2d, 2d' and 3a) and Iscove's Modified Dulbecco's Medium (Figures 2e, 2e' and 3b). Keeping in mind further degradation of diseased renal parenchyma and the lack of peritubular capillaries, only Leibovitz's L- 15 (Figures $2 \mathrm{f}, 2 \mathrm{f}$ and $3 \mathrm{c}$ ) and $\mathrm{CO}_{2}$ Independent Medium (Figures 2g, 2g' and 3d) appear as optimal candidates to compensate variations in $\mathrm{pH}$ of interstitial fluid and to stand the influence of harmful environment.

\section{Conclusion}

Present experiments illustrate that Leibovitz's L-15 (Figures 2f, $2 \mathrm{f}$ and $3 \mathrm{c}$ ) and $\mathrm{CO}_{2}$ Independent Medium (Figures $2 \mathrm{~g}, 2 \mathrm{~g}$ ' and $3 \mathrm{~d}$ ) appear suitable for the initial phase of implantation. Although both of the media exhibit the lowest content of glucose as compared to others (Table 1), they are able to maintain a constant $\mathrm{pH}$ independently from the actual $\mathrm{NaH}_{2} \mathrm{CO}_{3}$ and $\mathrm{CO}_{2}$ content in interstitial fluid of diseased parenchyma. The outcome of excellent morphology is a further argument to explore Leibovitz's L-15 and $\mathrm{CO}_{2}$ Independent Medium more intensively in ongoing experiments. However, even important is that in present experiments, no information is contained if both media exhibit a toxic influence on organ or systemic functions when implanted in the human organism.

\section{References}

1. Reule S, Gupta S (2011) Kidney regeneration and resident stem cells. Organogenesis 7: 135-139.

2. Harari-Steinberg O, Pleniceanu O, Dekel B (2011) Selecting the optimal cell for kidney regeneration: fetal, adult or reprogrammed stem cells. Organogenesis 7: 123-134.

3. Burst VR, Gillis M, Pütsch F, Herzog R, Fischer JH, et al. (2010) Poor cell survival limits the beneficial impact of mesenchymal stem cell transplantation on acute kidney injury. Nephron Exp Nephrol 114: e107-116.

4. Boor P, Floege J (2011) Chronic kidney disease growth factors in renal fibrosis. Clin Exp Pharmacol Physiol 38: 441-450.

5. Caldas HC, Fernandes IM, Gerbi F, Souza AC, Baptista MA, et al. (2008) Effect of whole bone marrow cell infusion in the progression of experimental chronic renal failure. Transplant Proc 40: 853-855

6. Bussolati B, Camussi G (2007) Stem cells in acute kidney injury. Contrib Nephrol 156: 250-258

7. Hammerman MR (2009) Xenotransplantation of pancreatic and kidney primordia-where do we stand? Transpl Immunol 21: 93-100.

8. Kim SS, Park HJ, Han J, Gwak SJ, Park MH, et al. (2007) Improvement of kidney failure with fetal kidney precursor cell transplantation. Transplantation 83: $1249-1258$

9. Ricardo SD, van Goor H, Eddy AA (2008) Macrophage diversity in renal injury and repair. J Clin Invest 118: 3522-3530.

10. Zoja C, Garcia PB, Remuzzi G (2009) The role of chemokines in progressive renal disease. Front Biosci 14: 1815-1822.

11. Liu Y (2011) Cellular and molecular mechanisms of renal fibrosis. Nat Rev Nephrol 7: 684-696.

12. Lee DW, Faubel S, Edelstein CL (2011) Cytokines in acute kidney injury (AKI). Clin Nephrol 76: 165-173.

13. Chung AC, Lan HY (2011) Chemokines in renal injury. J Am Soc Nephrol 22 802-809.

14. Chiang CK, Tanaka T, Nangaku M (2012) Dysregulated oxygen metabolism of the kidney by uremic toxins: review. J Ren Nutr 22: 77-80.

15. Palm F, Nordquist L (2011) Renal tubulointerstitial hypoxia: cause and consequence of kidney dysfunction. Clin Exp Pharmacol Physiol 38: 474-480.

16. Bonventre JV, Yang L (2011) Cellular pathophysiology of ischemic acute kidney injury. J Clin Invest 121: 4210-4221.

17. Miyamoto T, Carrero JJ, Stenvinkel P (2011) Inflammation as a risk factor and target for therapy in chronic kidney disease. Curr Opin Nephrol Hypertens 20: 662-668.

18. Roessger A, Denk L, Minuth WW (2009) Potential of stem/progenitor cell cultures within polyester fleeces to regenerate renal tubules. Biomaterials 30 : 3723-3732.

19. Minuth WW, Denk L, Glashauser A (2010) Cell and drug delivery therapeutics for controlled renal parenchyma regeneration. Adv Drug Deliv Rev 62: 841-854

20. Kuzma-Kuzniarska M, Rak-Raszewska A, Kenny S, Edgar D, Wilm B, et al. (2012) Integration potential of mouse and human bone marrow-derived mesenchymal stem cells. Differentiation 83: 128-137.

21. Blancas AA, Shih AJ, Lauer NE, McCloskey KE (2011) Endothelial cells from embryonic stem cells in a chemically defined medium. Stem Cells Dev 20: 2153-2161.

22. Goedecke A, Wobus M, Krech M, Münch N, Richter K, et al. (2011) Differentia effect of platelet-rich plasma and fetal calf serum on bone marrow-derived human mesenchymal stromal cells expanded in vitro. J Tissue Eng Regen Med $5: 648-654$.

23. Wang DH, Li FR, Zhang Y, Wang YQ, Yuan FH (2010) Conditioned medium from renal tubular epithelial cells stimulated by hypoxia influences rat bone marrow-derived endothelial progenitor cells. Ren Fail 32 : 863-870. 
Citation: Minuth WW, Denk L, Gruber M (2013) Search for Chemically Defined Culture Media to Assist Initial Regeneration of Diseased Renal Parenchyma after Stem/Progenitor Cell Implantation. J Tissue Sci Eng 4: 123. doi:10.4172/2157-7552.1000123

Page 7 of 7

24. Minuth WW, Denk L, Meese C, Rachel R, Roessger A (2009) Ultrastructural insights in the interface between generated renal tubules and a polyester interstitium. Langmuir 25: 4621-4627.

25. Minuth WW, Denk L, Miess C, Glashauser A (2011) Peculiarities of the extracellular matrix in the interstitium of the renal stem/progenitor cell niche. Histochem Cell Biol 136: 321-334.

26. Liu H, Liu S, Li Y, Wang X, Xue W, et al. (2012) The role of SDF-1-CXCR4/ CXCR7 axis in the therapeutic effects of hypoxia-preconditioned mesenchymal stem cells for renal ischemia/reperfusion injury. PLoS One 7: e34608.

27. Gao J, Liu R, Wu J, Liu Z, Li J, et al. (2012) The use of chitosan based hydrogel for enhancing the therapeutic benefits of adipose-derived MSCs for acute kidney injury. Biomaterials 33: 3673-3681.

28. Sedrakyan S, Da Sacco S, Milanesi A, Shiri L, Petrosyan A, et al. (2012) Injection of amniotic fluid stem cells delays progression of renal fibrosis. J Am Soc Nephrol 23: 661-673.

29. Grande MT, Pérez-Barriocanal F, López-Novoa JM (2010) Role of inflammation in túbulo-interstitial damage associated to obstructive nephropathy. J Inflamm (Lond) 7: 19.

30. Giuliani S, Perin L, Sedrakyan S, Kokorowski P, Jin D, et al. (2008) Ex vivo whole embryonic kidney culture: a novel method for research in development regeneration and transplantation. J Urol 179: 365-370. 